\title{
THE ITERATION OF RANDOM TESSELLATIONS AND A CONSTRUCTION OF A HOMOGENEOUS PROCESS OF CELL DIVISIONS
}

\author{
JOSEPH MECKE* AND \\ WERNER NAGEL, ${ }^{* * *}$ Friedrich-Schiller-Universität Jena \\ VIOLA WEISS, ${ }^{* * *}$ Fachhochschule Jena
}

\begin{abstract}
A random tessellation of $\mathbb{R}^{d}$ is said to be homogeneous if its distribution is invariant under all shifts of $\mathbb{R}^{d}$. The iteration of homogeneous random tessellations is described in a new manner that makes it evident that the resulting random tessellation is homogeneous again. Furthermore, a tessellation-valued process is constructed, the random states of which are homogeneous random tessellations stable under iteration (STIT). It can be interpreted as a process of subsequent division of cells.
\end{abstract}

Keywords: Stochastic geometry; random tessellation; tessellation-valued stochastic process; Poisson point process; iteration of tessellations; nesting of tessellations; stability of distributions

2000 Mathematics Subject Classification: Primary 60D05

Secondary 60G55

\section{Introduction}

In stochastic geometry the theory of point processes is widely and successfully applied. Often, ensembles of geometric objects can be considered to be realizations of point processes on appropriate state spaces. In particular, the theory of Poisson point processes is developed on general measurable spaces; see [4]. Thus, geometric objects, e.g. lines, segments, and balls, can be described by points in a parameter space [11] as well as being considered to be elements of a class of sets, e.g. polytopes and convex bodies (see [10]).

At least by methodical reasons, point processes on such complex state spaces are often reinterpreted as marked point processes. Many descriptions, e.g. of germ-grain-models or Boolean models, are based on a numbering of the points $x_{k}$ in a Euclidean space and an indexed family of random marks $M_{k}, k \in \mathbb{N}$, and the pairs $\left(x_{k}, M_{k}\right)$ describe the elements of a random ensemble of geometric objects.

On the one hand, this yields a more intuitive description of a model as an ensemble of points that are attached to sets or other marks expressing further properties. On the other hand, in many models a separated treatment of the points and of their marks is possible and allows well comprehensible results.

From a strict mathematical point of view, such a numbering method-which of course must be measurable - can cause problems when the homogeneity (i.e. spatial stationarity) of the model has to be shown. The methods of numbering which are usually applied refer to a fixed

Received 23 May 2007; revision received 17 September 2007.

* Postal address: Fakultät für Mathematik und Informatik, Friedrich-Schiller-Universität Jena, D-07737 Jena, Germany.

** Email address: nagel@minet.uni-jena.de

*** Postal address: Fachhochschule Jena, D-07703 Jena, Germany. 
origin of the Euclidean space, and this is not compatible with homogeneity. Therefore, for clear definitions and strict theoretical investigations, it is preferable to avoid numbering and to consider marked point processes as point processes on a product space; see, e.g. [10, Chapter 3].

The present paper is focused on certain procedures of cell division in random tessellations. In Sections 2 and 3 we deal with the operation of iteration of tessellations, also referred to as nesting. Roughly speaking, the operation of iteration means that, for a tessellation, which is also the 'frame tessellation', the cells are subdivided by other pairwise different tessellations. The authors first learned from R. V. Ambartzumian the idea of this operation in the 1980s; cf. [6], [8], and [9]. He also posed the problem of the existence of a limit for repeated iteration. Since that time, the operation was used and studied by several authors; see, e.g. the references in [8]. The usual way of defining the iteration of random tessellations is based on a numbering of the cells of the frame tessellation and then attaching independent and identically distributed (i.i.d.) random tessellations to these cells. Even if it is intuitively clear that this yields a homogeneous tessellation if all the ingredients are homogeneous, a strict proof of the homogeneity of the resulting random tessellation has been missing up to now. In the present paper the operation of iteration of random tessellations by independent random tessellations is defined in a new way that makes this homogeneity evident.

Finally, in Section 4, for random tessellations, a process of cell division by random hyperplanes is introduced. This construction can be understood as a modification of that model which is used in Section 2 for the iteration. In particular, the process of cell division is applied to tessellations that are stable with respect to iteration (STIT). This yields a Markov process, the states of which are STIT tessellations.

The definitions and results are presented for an arbitrary dimension $d \geq 2$. For a better intuition, we can always consider the particular case in which $d=2$.

\section{Iteration of tessellations}

Let $\mathbb{N}=\{1,2, \ldots\}$ denote the set of positive integers, let $\mathbb{R}^{d}$ denote the $d$-dimensional Euclidean space, and let $\Re_{d}$ denote the $\sigma$-algebra of Borel sets in $\mathbb{R}^{d}$.

\subsection{Random tessellations}

A tessellation is a locally finite partition of $\mathbb{R}^{d}$ into compact convex polytopes. Denote by int $Z$ the interior of a set $Z$ and denote by $\partial Z$ its boundary.

Definition 1. A tessellation $m$ in $\mathbb{R}^{d}$ is given by

$$
m=\bigcup_{Z \in C} \partial Z
$$

where $C$ is a countable set of compact convex polytopes with inner points and with the following properties.

(i) For all compact $B \subset \mathbb{R}^{d}$, the set $\{Z \in C: Z \cap B \neq \varnothing\}$ is finite.

(ii) For all $Z, Z^{\prime} \in C$, where $Z \neq Z^{\prime}$ holds, int $Z \cap$ int $Z^{\prime}=\varnothing$.

(iii) $\bigcup_{Z \in C} Z=\mathbb{R}^{d}$.

The elements $Z \in C$ are called the cells of the tessellation. From now on, we denote the set $C$ of polytopes related to a tessellation $m$ in the manner just described by $C(m)$. 
Denote by $\mathcal{M}_{\mathcal{F}}$ the set of all tessellations in $\mathbb{R}^{d}$. If $\mathcal{M}$ denotes the set of all countable sets of polytopes according to Definition 1 then (1) describes a one-to-one correspondence between $\mathcal{M}$ and $\mathcal{M}_{\mathcal{F}}$.

Furthermore, let $\mathcal{F}$ denote the set of all closed subsets of $\mathbb{R}^{d}$, and let $\mathfrak{F}$ denote the Effros $\sigma$-algebra (or Borel $\sigma$-algebra with respect to the topology of closed convergence) on $\mathcal{F}$; cf. [7, Chapter 1]. In Lemma 6.1.2 of [10] it was shown that $\mathcal{M}$ is a Borel subset of $\mathscr{F}\left(\mathcal{F}^{\prime}\right)$, which is the set of all closed subsets of the topological space $\mathcal{F}^{\prime}=\mathscr{F} \backslash\{\varnothing\}$. In the same lemma it was also stated that, for tessellations which fulfill the 'face-to-face' condition, the mapping

$$
\varphi_{d-1}: C(m) \rightarrow \varsigma_{d-1}(m)
$$

is measurable, where $s_{d-1}(Z)$ denotes the set of all $(d-1)$-faces of a polytope $Z$ and $s_{d-1}(m)=\bigcup_{Z \in C(m)} \S_{d-1}(Z)$. For the particular case of the $(d-1)$-faces of the polytopes, the proof of this lemma can also be applied to tessellations that do not necessarily have the 'face-to-face' property. (Note that these faces of the polytopes do not necessarily coincide with how the $(d-1)$-faces are defined for a tessellation.) Furthermore, in the proof of Satz 3.5.3 of [10] it was shown that the mapping

$$
\delta_{d-1}(m) \rightarrow \bigcup_{F \in \delta_{d-1}(m)} F
$$

is a measurable mapping into $[\mathcal{F}, \mathfrak{F}]$. Hence, the map $C(m) \rightarrow m$ described by (1) is also measurable. Thus, we have two measurable spaces for tessellations, namely $[\mathcal{M}, \mathfrak{M}]$ if we consider a tessellation as an ensemble of convex polytopes and $\left[\mathcal{M}_{\mathcal{F}}, \mathfrak{M}_{\mathcal{F}}\right]$ if we consider a tessellation as the closed set of the boundary points of the cells. We will switch between both descriptions arbitrarily.

A random tessellation is a random element $\Phi$ in the measurable space $\left[\mathcal{M}_{\mathcal{F}}, \mathfrak{M}_{\mathcal{F}}\right]$. Note that $\Phi$ is a random closed set (RACS) in the sense of Matheron; see [3] and [7]. For brevity, we sometimes also say that the random element $C(\Phi)$ in the measurable space $[\mathcal{M}, \mathfrak{M}]$ is a random tessellation.

A random tessellation can also be described as a point process on the set of convex polytopes; see [10]. Furthermore, such a point process can also be given as a marked point process with reference points in the Euclidean space $\mathbb{R}^{d}$ and polytopes as marks.

For $x \in \mathbb{R}^{d}$, denote by $T_{x}$ the translation of $\mathbb{R}^{d}$ by $-x$, and this symbol is also used for translations of tessellations, i.e. $T_{x}: \mathcal{M}_{\mathcal{F}} \rightarrow \mathcal{M}_{\mathcal{F}}$ with $T_{x} \varphi=\varphi-x, \varphi \in \mathcal{M}_{\mathcal{F}}$, and for $T_{x}: \mathcal{M} \rightarrow \mathcal{M}$ analogously. If $\Phi$ is a random tessellation with distribution $\mathrm{P}$ then the distribution of $T_{x} \Phi$ is denoted by $T_{x} \mathrm{P}$.

A random tessellation is said to be homogeneous (i.e. spatially stationary) if its distribution is invariant under translations. Accordingly, a distribution $\mathrm{P}$ on $\left[\mathcal{M}_{\mathcal{F}}, \mathfrak{M}_{\mathcal{F}}\right]$ is called homogeneous if $\mathrm{P}=T_{x} \mathrm{P}$ for all $x \in \mathbb{R}^{d}$.

\subsection{Definition of iteration}

The iteration, also referred to as nesting, is an operation where the cells of a tessellation $m_{0}$ are subdivided by a sequence $\left(m_{k}\right)_{k \in \mathbb{N}}$ of other tessellations. For different cells of $m_{0}$, different tessellations of the sequence are used.

Using a numbering for the cells of $m_{0}$ such that $C\left(m_{0}\right)=\left\{Z_{1}, Z_{2}, \ldots\right\}$, the set of boundary points of the iterated tessellation can be defined as

$$
\tau\left(m_{0},\left(m_{k}\right)_{k}\right)=m_{0} \cup \bigcup_{k \in \mathbb{N}}\left(Z_{k} \cap m_{k}\right) .
$$


Equivalently, the set of cells of the iterated tessellation is given by

$$
C\left(\mathcal{I}\left(m_{0},\left(m_{k}\right)_{k}\right)\right)=\bigcup_{k \in \mathbb{N}}\left\{Z_{k} \cap Z_{k j}: j \in \mathbb{N}, \text { int } Z_{k} \cap \text { int } Z_{k j} \neq \varnothing\right\},
$$

where the $Z_{k j}, j \in \mathbb{N}$, are the cells of the tessellation $m_{k}$. Thus, the iteration is an operation

$$
\tau: \mathcal{M}_{\mathcal{F}} \times \mathcal{M}_{\mathcal{F}}^{\mathbb{N}} \rightarrow \mathcal{M}_{\mathcal{F}},
$$

where $\mathcal{M}_{\mathcal{F}}{ }^{\mathbb{N}}$ denotes the set of all sequences $\left(m_{k}\right)_{k \in \mathbb{N}}$ of tessellations $m_{k} \in \mathcal{M}_{\mathcal{F}}$. In this context the tessellation $m_{0}$ is called the frame tessellation.

The iteration can be applied to random tessellations $\Phi_{0},\left(\Phi_{k}\right)_{k \in \mathbb{N}}$ as well. In order to generate a random tessellation, the method of numbering the cells has to be measurable.

If $\Phi_{0}$ and all the $\Phi_{k}$ are homogeneous and independent and if, additionally, the $\Phi_{k}$ are identically distributed, then it seems to be intuitively clear that $\mathcal{L}\left(\Phi_{0},\left(\Phi_{k}\right)_{k}\right)$ is homogeneous. But, owing to the numbering, it is not straightforward to prove it formally. This is a motivation to provide an alternative description for the iteration of tessellations.

\subsection{Iteration of homogeneous random tessellations}

The purpose of the following construction is a definition of the iteration such that the independence of the ingredient tessellations on the one hand and the homogeneity of the resulting tessellation on the other hand become evident.

Let $\mathbb{R}_{-}=(-\infty, 0)$, let $\Re_{-}=\mathfrak{R} \cap \mathbb{R}_{-}$, and let $\ell$ and $\ell_{-}$be the Lebesgue measures on $\mathbb{R}$ and $\mathbb{R}_{-}$, respectively. The Lebesgue measure on $\mathbb{R}^{d}$ is denoted by $\ell_{d}$.

Let $\Xi$ be a Poisson point process on the measurable space

$$
\left[\mathbb{R}^{d} \times \mathbb{R}_{-} \times \mathcal{M}_{\mathcal{F}}, \mathfrak{R}_{d} \otimes \mathfrak{R}_{-} \otimes \mathfrak{M}_{\mathcal{F}}\right]
$$

with intensity measure $\ell_{d} \times \ell_{-} \times \mathrm{Q}$, where $\mathrm{Q}$ is a probability measure on $\left[\mathcal{M}_{\mathcal{F}}, \mathfrak{M}_{\mathcal{F}}\right]$. Poisson point processes with $\sigma$-finite intensity measures on measurable spaces were constructed, for example, in [4].

For $(x, r, \psi) \in \mathbb{R}^{d} \times \mathbb{R}_{-} \times \mathcal{M}_{\mathcal{F}}$, we interpret $x$ as the reference point and $r$ as the priority of the tessellation $\psi$. For a compact convex set $Z \subset \mathbb{R}^{d}$ with interior int $Z \neq \varnothing$, we define the random variables

$$
R(Z)=\max \left\{r \in \mathbb{R}_{-}:(x, r, \psi) \in \Xi, x \in \operatorname{int} Z\right\},
$$

$\xi(Z)$, and $\Psi(Z)$ by

$$
(\xi(Z), R(Z), \Psi(Z)) \in \Xi .
$$

This means that $\Psi(Z)$ is the random tessellation that has the highest priority among all the tessellations with their reference points in the interior of $Z$. Note that, owing to the properties of the Poisson point process $\Xi$ and the compactness of $Z$, the number $R(Z)$ almost surely (a.s.) exists and that $\Psi(Z)$ is a.s. uniquely defined.

Remark. The Poisson process $\Xi$ on the phase space $\mathbb{R}^{d} \times \mathbb{R}_{-} \times \mathcal{M}_{\mathcal{F}}$ may be reinterpreted as a marked point process with phase space $\mathbb{R}^{d} \times \mathbb{R}_{-}$and mark space $\mathcal{M}_{\mathcal{F}}$ of tessellations. The points in $\mathbb{R}^{d} \times \mathbb{R}_{-}$form a Poisson point process with intensity measure $\ell_{d} \times \ell_{-}$, and the marks are i.i.d. in $\mathcal{M}_{\mathcal{F}}$ according to the probability measure $\mathrm{Q}$ and independent of the Poisson process on $\mathbb{R}^{d} \times \mathbb{R}_{-}$. 
In Definition 2, below, the tessellations are understood to be the closed sets of boundaries of the cells.

Definition 2. Let $\Phi$ be a homogeneous random tessellation of $\mathbb{R}^{d}$ with distribution $\mathrm{P}$, and let $\Xi$ be a Poisson point process on $\mathbb{R}^{d} \times \mathbb{R}_{-} \times \mathcal{M}_{\mathcal{F}}$ with intensity measure $\ell_{d} \times \ell_{-} \times \mathrm{Q}$. The iteration of $\Phi$ by $\Xi$ is defined as

$$
\Phi \odot \Xi=\Phi \cup \bigcup_{Z \in C(\Phi)}(Z \cap \Psi(Z)) .
$$

This means that into any cell $Z$ of the tessellation $\Phi$, the cut-out of $\Psi(Z)$ is nested. In the context of this definition we refer to $\Phi$ as the frame tessellation.

If $\Phi$ and $\Xi$ are independent, the distribution of $\Phi \odot \Xi$ is denoted by $\mathrm{P} \boxplus \mathrm{Q}$.

We note that our approach is also suitable for studying repeated iteration as well as random processes on the state space of tessellations where the transition is given by iteration.

\section{Properties}

Owing to the properties of the Poisson process $\Xi$ introduced above, the following assertions are evident. This illustrates that the chosen approach proves to be efficient.

Lemma 1. Let $\Phi$ be a homogeneous random tessellation, let $\Xi$ be the Poisson point process defined above with intensitymeasure $\ell_{d} \times \ell_{-} \times \mathrm{Q}$, and let $\Phi$ and $\Xi$ be independent. Furthermore, assume that $\left(\Psi_{k}\right)_{k \in \mathbb{N}}$ is a sequence of i.i.d. random tessellations with distribution $\mathrm{Q}$, also independent of $\Phi$. Then the tessellation $\Phi \odot \Xi$ and the result of iteration with an arbitrary measurable numbering of the cells of $\Phi$, i.e. $\mathcal{L}\left(\Phi,\left(\Psi_{k}\right)_{k}\right)$, are identically distributed RACSs.

The crucial argument is that in the Poisson point process $\Xi$ the marks $\Psi(Z)$ with respect to any measurable numbering of the cells are i.i.d. random variables with distribution $\mathrm{Q}$.

The symbol $T_{x}$ that was introduced above for the translation in $\mathbb{R}^{d}$ by $-x$ and for tessellations will also be applied to $\Xi$ as

$$
T_{x} \Xi=\left\{\left(y-x, r, T_{x} \psi\right):(y, r, \psi) \in \Xi\right\} .
$$

The translation invariance of the Lebesgue measure $\ell_{d}$ immediately yields the following assertion.

Lemma 2. Let $\Xi$ be the Poisson point process defined above, and let $x \in \mathbb{R}^{d}$. Then $T_{x} \Xi$ is a Poisson point process with intensity measure $\ell_{d} \times \ell_{-} \times T_{x} \mathrm{Q}$, where $T_{x} \mathrm{Q}$ denotes the image of the measure $\mathrm{Q}$ under the translation $T_{x}$.

Hence, the translation $T_{x}$ commutes with the iteration, ' $\odot$ ', and Theorem 1, below, becomes evident.

More formally, Lemma 2 implies that, for all $x \in \mathbb{R}^{d}$,

$$
T_{x}(\Phi \odot \Xi)=T_{x} \Phi \odot T_{x} \Xi
$$

and, thus,

$$
T_{x}(\mathrm{P} \boxplus \mathrm{Q})=T_{x} \mathrm{P} \boxplus T_{x} \mathrm{Q}
$$

for all distributions $\mathrm{P}$ and $\mathrm{Q}$ on $\left[\mathcal{M}_{\mathcal{F}}, \mathfrak{M}_{\mathcal{F}}\right]$. Hence, if both $\mathrm{P}$ and $\mathrm{Q}$ are homogeneous distributions on $\left[\mathcal{M}_{\mathcal{F}}, \mathfrak{M}_{\mathcal{F}}\right]$ then $\mathrm{P} \boxplus \mathrm{Q}$ is also homogeneous. 


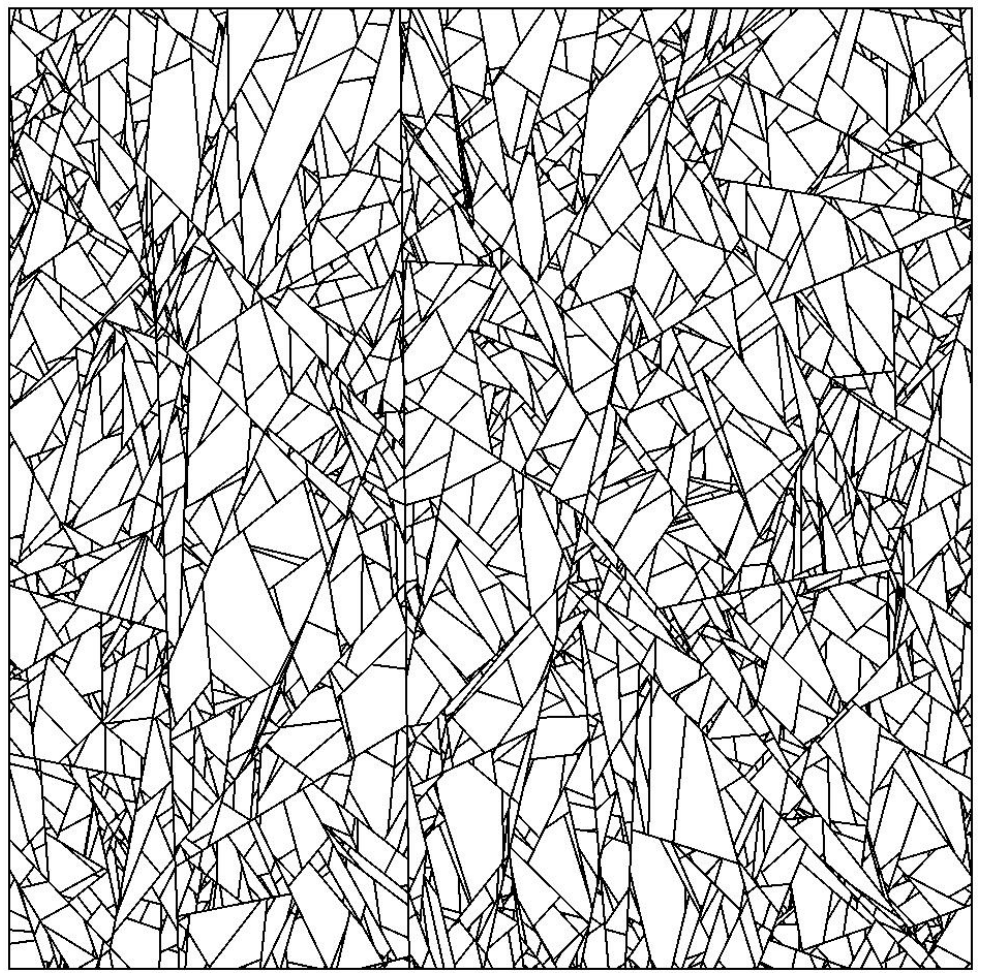

FIGURE 1: Simulation of a planar homogeneous and anisotropic STIT tessellation. (Provided by Joachim Ohser, Darmstadt.)

Theorem 1. Let $\Phi$ be a homogeneous tessellation, and let $\Xi$ be a Poisson point process on $\mathbb{R}^{d} \times \mathbb{R}_{-} \times \mathcal{M}_{\mathcal{F}}$ with intensity measure $\ell_{d} \times \ell_{-} \times \mathrm{Q}$. If the distribution $\mathrm{Q}$ is homogeneous and $\Phi$ and $\Xi$ are independent then $\Phi \odot \Xi$ is also a homogeneous tessellation.

In order to define the stability under iteration of tessellations for distributions of homogeneous random tessellations, we use the notation $\mathrm{P}(r \cdot)$ for the distribution of the homothetic random tessellation $r \cdot \Phi, r \in \mathbb{R}_{+}=(0, \infty)$, if $\mathrm{P}$ is the distribution of $\Phi$. Formally, for $\varphi \in \mathcal{M}_{\mathcal{F}}$ and $A \in \mathfrak{M}_{\mathcal{F}}$, we define $r \varphi=\{r x: x \in \varphi\}$ and $r A=\{r \varphi: \varphi \in A\}$.

Definition 3. A homogeneous random tessellation $\Phi$ with distribution P is called STIT if, for any integer $n>1$,

$$
\mathrm{P}_{n}(\cdot)=\mathrm{P}(n \cdot)
$$

where the $\mathrm{P}_{2}, \mathrm{P}_{3}, \ldots$ are recursively defined by $\mathrm{P}_{2}=\mathrm{P} \boxplus \mathrm{P}$ and $\mathrm{P}_{n+1}=\mathrm{P}_{n} \boxplus \mathrm{P}$ for $n=$ $2,3, \ldots$

Some basic results on random homogeneous STIT tessellations can be found in [9] and a global construction is described in [5]. Figure 1 shows a simulation of a planar homogeneous and anisotropic STIT tessellation. 


\section{A process of cell division in tessellations}

In the previous section the process $\Xi$ was used for a definition of the iteration of tessellations. This process is now modified in order to describe a process of consecutive cell division. This means that, for each cell of a tessellation, there is a random time when it is divided by a single random hyperplane. Formally, the difference is that instead of the Poisson point process $\Xi$ on $\mathbb{R}^{d} \times \mathbb{R}_{-} \times \mathcal{M}_{\mathcal{F}}$, we will now use a Poisson point process $\Sigma$ on $\mathbb{R}^{d} \times \mathbb{R}_{-} \times \mathcal{N}$, where $\mathcal{N}$ describes births of hyperplanes that can under some conditions divide an existing cell.

This substitution of tessellations by a sequence of hyperplanes, marked with birth times, still allows an intuitive interpretation related to iteration in the following sense. If we consider $n$-fold repeated iterations and a limit for $n$ tending to $\infty$ then a rescaling with the factor $n$ of the tessellations is necessary in order to obtain convergence to a tessellation. Thus, for large rescaling factors of the tessellations that are nested into the cells of a frame tessellation, most of the cut-outs to be nested in are either empty or consist of one hyperplane that divides a cell of the frame tessellation. This is somehow reflected formally in the following model.

At the end of this section, this approach is applied to tessellations that are STIT. This shows how the construction of STIT tessellations is closely related to these processes of cell division.

\subsection{A homogeneous birth process of hyperplanes}

Let $\mathbb{R}_{+}=(0, \infty)$, let $\Re_{+}=\mathfrak{R} \cap \mathbb{R}_{+}$, and let $\ell_{+}$be the Lebesgue measure on $\mathbb{R}_{+}$. Let $\mathscr{H}$ denote the set of hyperplanes in $\mathbb{R}^{d}$, and let $\mathfrak{H}$ denote the $\sigma$-algebra on $\mathscr{H}$ that is induced by the Effros $\sigma$-algebra on the set $\mathcal{F}$ (cf. [10]). Furthermore, assume that $v$ is a nonvanishing translation invariant locally finite measure on $[\mathscr{H}, \mathfrak{H}]$. The measure $v$ determines the so-called directional distribution $\vartheta$ which lives on the space of directions, i.e. the set of lines through the origin. It is required that $\vartheta$ is not concentrated on a set of lines which lie in one single hyperplane.

Now let $\Pi$ be a Poisson point process on the measurable space $\left[\mathcal{H} \times \mathbb{R}_{+}, \mathfrak{H} \otimes \Re_{+}\right]$with intensity measure $v \times \ell_{+}$. The interpretation of a point $(h, s) \in \mathscr{H} \times \mathbb{R}_{+}$that appears in $\Pi$ is that at time $s>0$, the hyperplane $h$ is born. Denote by $\mathcal{N}$ the set of all countable subsets $N$ of $\mathscr{H} \times \mathbb{R}_{+}$with \# $\left(N \cap\left[C_{1}\right] \times C_{2}\right)<\infty$ for bounded $C_{1} \subset \mathbb{R}^{d}$ and bounded $C_{2} \subset \mathbb{R}_{+}$, where $\left[C_{1}\right]=\left\{h \in \mathscr{H}: h \cap C_{1} \neq \varnothing\right\}$ and \# denotes the number of elements of a set. Let $\mathfrak{N}$ denote the $\sigma$-algebra on $\mathcal{N}$ that is generated by

$$
\left\{\{N \subset \mathcal{N}: \#(N \cap B)=k\}: k \in \mathbb{N} \cup\{0\}, B \in \mathfrak{H} \otimes \mathfrak{R}_{+}\right\} .
$$

The distribution of $\Pi$ is denoted by $S$, which is a probability measure on $[\mathcal{N}, \mathfrak{N}]$.

For any $t>0$, we define the hyperplane process $\Gamma_{t}$ of those hyperplanes from $\Pi$ that are born before time $t$, i.e. $\Gamma_{t}=\{h:(h, s) \in \Pi, s<t\}$. For all $t>0$, this is a homogeneous Poisson hyperplane process. Thus, we obtain a random process $\left(\Gamma_{t}\right)_{t>0}$ on the state space of hyperplane ensembles. It has the property that $\Gamma_{t_{1}} \subseteq \Gamma_{t_{2}}$ for $t_{1}<t_{2}$. This process will be referred to as a birth process of hyperplanes.

The process $\{(h, s) \in \Pi: s<t\}$ can also be understood as a marked point process of hyperplanes with the birth times as marks. These marks have a uniform distribution on $(0, t)$.

\subsection{A random ensemble of birth processes of hyperplanes}

In the next preparatory step we introduce $\Sigma$ as a Poisson point process on the measurable space

$$
\left[\mathbb{R}^{d} \times \mathbb{R}_{-} \times \mathcal{N}, \mathfrak{R}_{d} \otimes \mathfrak{R}_{-} \otimes \mathfrak{N}\right]
$$

with intensity measure $\ell_{d} \times \ell_{-} \times S$, where $S$ is the distribution of the process $\Pi$ of Subsection 4.1 . 
The point process $\Sigma$ can be understood as a marked Poisson point process on $\mathbb{R}^{d} \times \mathbb{R}$ and the marks are from $\mathcal{N}$ with mark distribution S. Owing to the relation between $\Pi$ and $\left(\Gamma_{t}\right)_{t>0}$, the point process $\Sigma$ can also be interpreted as a random system of birth processes of hyperplanes where any of these birth processes is provided with a reference point in $\mathbb{R}^{d}$ and with a priority in $(-\infty, 0)$.

The Poisson point process $\Sigma$ has the following properties.

(i) For any $r<0$, the set $\left\{x:\left(x, r^{\prime}, \chi\right) \in \Sigma, r<r^{\prime}<0\right\}$ of those reference points with a priority greater than $r$ forms a point process; it is a homogeneous Poisson point process on $\mathbb{R}^{d}$ with finite intensity $|r|$.

(ii) The set of all reference points $\{x:(x, r, \chi) \in \Sigma\}$ is a.s. countably infinite and dense in $\mathbb{R}^{d}$.

(iii) Let $Z$ be a Borel subset of $\mathbb{R}^{d}$ with $0<\ell_{d}(Z)<\infty$. Then the set of all priorities of points in $\Sigma$ with the reference point in $Z$, i.e. the set $\left\{r \in \mathbb{R}_{-}:(x, r, \chi) \in \Sigma, x \in\right.$ int $\left.Z\right\}$, is a homogeneous Poisson point process on $(-\infty, 0)$ with finite intensity $\ell_{d}(Z)$.

(iv) From the Poisson point process $\Sigma$ with phase space $\mathbb{R}^{d} \times \mathbb{R}_{-} \times \mathcal{N}$ we obtain a point process $\tilde{\Sigma}$ with phase space $\mathbb{R}^{d} \times \mathbb{R}_{-} \times \mathcal{H} \times \mathbb{R}_{+}$as

$$
\tilde{\Sigma}=\{(x, r, h, s):(x, r, \chi) \in \Sigma,(h, s) \in \chi\} .
$$

Note that the operation which maps a realization of $\Sigma$ to a realization of $\tilde{\Sigma}$ is measurable with respect to the $\sigma$-algebras involved.

The point process $\tilde{\Sigma}$ is no longer a Poisson process but a Cox process with a random directing measure $\Theta$ on $\mathbb{R}^{d} \times \mathbb{R}_{-} \times \mathcal{H} \times \mathbb{R}_{+}$that can be constructed as follows. For given $x \in \mathbb{R}^{d}$ and $r<0$, denote by $\alpha_{x, r}$ the measure on $\mathbb{R}^{d} \times \mathbb{R}_{-} \times \mathcal{H} \times \mathbb{R}_{+}$which is induced by the measure $v \times \ell_{+}$and the map

$$
(h, s) \rightarrow(x, r, h, s)
$$

from $\mathscr{H} \times \mathbb{R}_{+}$into $\mathbb{R}^{d} \times \mathbb{R}_{-} \times \mathcal{H} \times \mathbb{R}_{+}$. Let $A$ be a Poisson process on $\mathbb{R}^{d} \times \mathbb{R}_{-}$with intensity measure $\ell_{d} \times \ell_{-}$. Then

$$
\Theta=\sum_{(x, r) \in A} \alpha_{x, r} .
$$

A point $(x, r, h, s) \in \tilde{\Sigma}$ corresponds to a hyperplane $h$ with birth time $s>0$, reference point $x \in \mathbb{R}^{d}$, and priority $r<0$. The phase space $\mathbb{R}^{d} \times \mathbb{R}_{-} \times \mathscr{H} \times \mathbb{R}_{+}$can be regarded as a second-countable locally compact Hausdorff space. Hence, we obtain the standard framework of random measures and Cox point processes in the sense of Kallenberg; see [2, Chapter 10] and the references therein.

In the following paragraphs the Cox process $\tilde{\Sigma}$ on a familiar phase space could be used instead of the Poisson process $\Sigma$ on a much more complicated phase space. However, this is more or less a matter of taste.

\subsection{A random division of a cell}

We start with the consideration of a pair $(Z, s)$, where $Z \subset \mathbb{R}^{d}$ is a compact convex polytope and $s>0$ is said to be the birth time of $Z$. Define the random variable $R(Z)$ by

$$
R(Z)=\max \{r:(x, r, \chi) \in \Sigma, x \in \text { int } Z\} .
$$


The a.s. existence and uniqueness of $R(Z)$ follows from (iii) in Subsection 4.2. Let $(\xi(Z), R(Z)$, $\chi(Z))$ be the unique point $(x, r, \chi) \in \Sigma$ with $x \in$ int $Z$ and $r=R(Z)$.

Next, choose $h_{Z}$ according to the conditions

$$
\left(h_{Z}, s_{Z}\right) \in \chi(Z)
$$

and

$$
s_{Z}=\min \left\{s^{\prime}:\left(h, s^{\prime}\right) \in \chi(Z), s^{\prime}>s, h \cap \operatorname{int} Z \neq \varnothing\right\} .
$$

The a.s. existence of $s_{Z}$ is guaranteed since the birth times of those lines in $\chi(Z)$ that hit $Z$ form a homogeneous Poisson process on $\mathbb{R}_{+}$with a positive and finite intensity (which depends on $Z$ and the directional distribution $\vartheta$ ).

This means that, from $\chi(Z)$, the hyperplane which is born first after the birth time $s$ of $Z$ is chosen. This hyperplane is now used to divide the cell $Z$. Denoting by $h^{+}$and $h^{-}$the two closed half-spaces generated by the hyperplane $h$, we obtain

$$
Z^{+}=Z \cap h_{Z}^{+}, \quad Z^{-}=Z \cap h_{Z}^{-},
$$

and both the new cells are provided with the birth time $s_{Z}$. Denote by $h(Z)=Z \cap h_{Z}$ the part of the hyperplane that appears in $Z$; for $d=2$, this is a chord.

In the next step the cells $Z^{+}$and $Z^{-}$are treated in the same way as $Z$ was treated before. It is essential that the birth times of the hyperplanes that are used for further division are greater than $s_{Z}$.

This procedure of consecutive division of cells can be continued ad infinitum. For any time $t>s$, denote by $G_{s, t}(Z)$ the union of all $(d-1)$-polytopes $h(\tilde{Z})$ in cells $\tilde{Z}$ which are generated by this procedure of consecutive division of $Z$ and which have a birth time in the interval $(s, t)$.

\subsection{A homogeneous process of cell division in a tessellation}

Now, for $\varepsilon>0$, let $\Phi_{\varepsilon}$ be a homogeneous random tessellation, regarded as a RACS, with hypersurface intensity $S_{V}=\varepsilon$ and directional distribution $\vartheta$. All the cells of $\Phi_{\varepsilon}$ are provided with the birth time $\varepsilon$. Let $\Sigma$ be a Poisson point process on $\mathbb{R}^{d} \times \mathbb{R}_{-} \times \mathcal{N}$ with intensity measure $\ell_{d} \times \ell_{-} \times \mathrm{S}$, where $\mathrm{S}$ is the probability measure on $[\mathcal{N}, \mathfrak{N}]$ which was introduced in Subsection 4.1 and, hence, is determined by the directional distribution $\vartheta$. Assume that $\Phi_{\varepsilon}$ and $\Sigma$ are independent.

Now the cells $Z$ of $\Phi_{\varepsilon}$ are divided as described above and, thus, for $t>\varepsilon$, the sets $G_{\varepsilon, t}(Z)$ are generated by $\Sigma$. We define the tessellation

$$
\Phi_{t}=\Phi_{\varepsilon} \cup \bigcup_{Z \in \Phi_{\varepsilon}} G_{\varepsilon, t}(Z)
$$

and, thus, we also define a random process $\left(\Phi_{t}\right)_{t>\varepsilon}$ on the state space of tessellations.

For any $t>\varepsilon$, the random tessellation $\Phi_{t}$ is homogeneous and has the hypersurface intensity $S_{V}=t$ (cf. Lemma 3, below, and [9]) and directional distribution $\vartheta$.

\subsection{Results for STIT tessellations}

Now we apply the process of cell division to homogeneous random tessellations that are STIT. Let $\stackrel{\text { D, }}{=}$ denote the identity of distributions. 
From the construction described above, the following assertions are in evidence.

Lemma 3. As in Subsection 4.3, let $Z$ be a compact convex polytope. Then, for $t>\varepsilon>0$, the set $G_{\varepsilon, t}(Z)$ has-up to a constant factor for the intensity-the same distribution as the construction of a STIT tessellation in a bounded window $Z$ in time $t-\varepsilon$ and with directional distribution $\vartheta$, as it was described in [9] and there denoted as $Y(t-\varepsilon, Z)$.

Thus, with the results in [9] and the notation introduced in Subsection 2.3, the following assertion is evident.

Theorem 2. Let $\Phi_{\varepsilon}$ be a homogeneous STIT tessellation with hypersurface intensity $S_{V}=\varepsilon$ and directional distribution $\vartheta$. Furthermore, let $\Sigma$ be a Poisson point process on $\mathbb{R}^{d} \times \mathbb{R}_{-} \times \mathcal{N}$ with intensity measure $\ell_{d} \times \ell_{-} \times \mathrm{S}$, where $\mathrm{S}$ is the probability measure on $[\mathcal{N}, \mathfrak{N}]$ that was introduced in Subsection 4.1, and let $\Phi_{\varepsilon}$ and $\Sigma$ be independent. Then the process $\left(\Phi_{t}\right)_{t \geq \varepsilon}$ that is defined by (2) has the following properties.

(i) For any $t>\varepsilon$, the tessellation $\Phi_{t}$ is a homogeneous STIT tessellation with hypersurface intensity $t$ and directional distribution $\vartheta$.

(ii) For $n \in \mathbb{N}$ and $\varepsilon \leq s_{1}<\cdots<s_{n}$, the distribution of $\left(\Phi_{s_{1}}, \ldots, \Phi_{s_{n}}\right)$ is invariant under all translations of $\mathbb{R}^{d}$.

(iii) If $\varepsilon \leq s<t$ then $\Phi_{s} \subseteq \Phi_{t}$, i.e. the set of boundary points of the cells of $\Phi_{s}$ is a subset of that one for $\Phi_{t}$.

(iv) The random process $\left(\Phi_{t}\right)_{t>\varepsilon}$ has the Markov property.

(v) For $\varepsilon \leq s<t$, let $\Xi_{t-s}$ be a Poisson point process on the space $\mathbb{R}^{d} \times \mathbb{R}_{-} \times \mathcal{M}_{\mathcal{F}}$ with intensity measure $\ell_{d} \times \ell_{-} \times \mathrm{Q}$, as in Section 2 , where here it is assumed that $\mathrm{Q}$ is the distribution of a homogeneous STIT tessellation with hypersurface density $t-s$ and directional distribution $\vartheta$. Then

$$
\Phi_{t} \stackrel{\mathrm{D}}{=} \Phi_{s} \odot \Xi_{t-s}
$$

Thus, if $\Phi_{\varepsilon}$ is a homogeneous STIT tessellation then the random process $\left(\Phi_{t}\right)_{t \geq \varepsilon}$ that is defined by (2) can be referred to as a random STIT process of cell division.

There are relations to fragmentation processes in the sense of [1] that are explained in [5].

\section{Acknowledgements}

We thank Professor Joachim Ohser, Hochschule Darmstadt, for providing the simulation in Figure 1. The authors are grateful for the comments of the Editor and of an anonymous referee.

\section{References}

[1] Bertoin, J. (2006). Random Fragmentation and Coagulation Processes. Cambridge University Press.

[2] Kallenberg, O. (1997). Foundations of Modern Probability. Springer, New York.

[3] Matheron, G. (1975). Random Sets and Integral Geometry. John Wiley, New York, London.

[4] Mecke, J. (1967). Stationäre zufällige Masse auf lokalkompakten Abelschen Gruppen. Z. Wahrscheinlichkeitsth. 9, 36-58.

[5] Mecke, J., Nagel, W. ANd Weiss, V. (2008). A global construction of homogeneous random planar tessellations that are stable under iteration. Stochastics Internat. J. Prob. Stoch. Process. 80, 51-67.

[6] Mecke, J., Nagel, W. ANd Weiss, V. (2007). Length distributions of edges of planar stationary and isotropic STIT tessellations. J. Contemp. Math. Anal. 42, 28-43.

[7] Molchanov, I. S. (2005). Theory of Random Sets. Springer, London. 
[8] Nagel, W. and Weiss, V. (2003). Limits of sequences of stationary planar tessellations. Adv. Appl. Prob. 35, 123-138.

[9] NAGEL, W. AND WEISs, V. (2005). Crack STIT tessellations: characterization of stationary random tessellations stable with respect to iteration. Adv. Appl. Prob. 37, 859-883.

[10] Schneider, R. AND WeIL, W. (2000). Stochastische Geometrie. Teubner, Stuttgart.

[11] Stoyan, D., Kendall, W. S. And Mecke, J. (1995). Stochastic Geometry and Its Applications, 2nd edn. John Wiley, Chichester. 